\title{
BEST POSSIBLE COMPACTNESS RESULTS OF LIONS-PEETRE TYPE
}

\author{
FERNANDO COBOS $^{1}$, MICHAEL CWIKEL $^{2}$ AND PEDRO MATOS ${ }^{1,3}$ \\ ${ }^{1}$ Departamento de Análisis Matemático, Facultad de Matemáticas, \\ Universidad Complutense de Madrid, 28040 Madrid, Spain \\ (cobos@eucmax.sim.ucm.es) \\ ${ }^{2}$ Department of Mathematics, Technion-Israel Institute of Technology, \\ Haifa 32000, Israel (mcwikel@math.technion.ac.il) \\ ${ }^{3}$ ESTG, Instituto Politécnico de Leiria, 2400 Leiria, \\ Portugal (matos@estg.iplei.pt)
}

(Received 12 November 1998)

\begin{abstract}
If $T: A_{0} \rightarrow B$ boundedly and $T: A_{1} \rightarrow B$ compactly, then a result of Lions-Peetre shows that $T: A \rightarrow B$ compactly for a certain class of spaces $A$ which are intermediate with respect to $A_{0}$ and $A_{1}$. We investigate to what extent such results can hold for arbitrary intermediate spaces $A$. The 'dual' case of an operator $S$ such that $S: X \rightarrow Y_{0}$ boundedly and $S: X \rightarrow Y_{1}$ compactly, is also considered, as well as similar questions for other closed operator ideals.
\end{abstract}

Keywords: interpolation of compact operators; rank-one interpolation spaces; general intermediate spaces; operator ideals

AMS 2000 Mathematics subject classification: Primary 46B70; 47D50

\section{Introduction}

This paper deals with compactness results for interpolated operators in the style of those established by Lions and Peetre [14] in 1964 (see also the lecture by Gagliardo [10]). Lions and Peetre deal with two situations. One of these is the case of a linear operator $T$ which is a bounded map of both of the spaces $A_{0}$ and $A_{1}$ of a Banach couple $\bar{A}=\left(A_{0}, A_{1}\right)$ into the same Banach space $B$ with the additional assumption that $T: A_{1} \rightarrow B$ is compact. The other is the 'dual' case of an operator $T$ which maps a given Banach space $A$ boundedly into both of the spaces of a Banach couple $\bar{B}=\left(B_{0}, B_{1}\right)$, and here it is always assumed that $T: A \rightarrow B_{1}$ is compact. In the first case, Lions and Peetre show that if $A$ is a space of the class $\mathcal{C}_{K}(\theta, \bar{A})$ for some $\theta \in(0,1)$, then $T: A \rightarrow B$ is also compact. In the 'dual' case, they prove that $T: A \rightarrow B$ is compact for each space $B$ of the class $\mathcal{C}_{J}(\theta, \bar{B})$. (We shall recall the meaning of the notation used here in $\S 2$.)

Compactness results of Lions-Peetre type have interesting applications to function spaces (for example, to Sobolev spaces). Several authors have also investigated them 
from other points of view. For example, a quantitative version of Lions-Peetre results in terms of entropy numbers can be found in the book by Pietsch [17, Propositions 12.1.11 and 12.1.12]. Similar results in terms of the measure of non-compactness were established by Edmunds and Teixeira [20]. More abstract versions that work for injective surjective closed operator ideals are due to Heinrich [12, Propositions 1.6 and 1.7].

Lions-Peetre type compactness results are also important tools for establishing a number of other compactness theorems in interpolation theory (see, for example, the papers by Persson [16], Cobos and Peetre [7], and Cobos, Kühn and Schonbek [5]).

Just as in [14], also in the other corresponding results mentioned above (see [17], [20] and [12]), the spaces $A$ and $B$ are required to satisfy $A \in \mathcal{C}_{K}(\theta, \bar{A})$ or $B \in \mathcal{C}_{J}(\theta, \bar{B})$. These are, in fact, rather strong restrictions on $A$ and $B$. It has been pointed out by Mastyło (see $[\mathbf{1 5}$, Theorem 1]) that the Lions-Peetre results hold under weaker conditions than these, i.e. the compactness of $T: A \rightarrow B$ can also be proved when $A$ or $B$, respectively, belong to other classes which generalize $\mathcal{C}_{K}(\theta, \bar{A})$ or $\mathcal{C}_{J}(\theta, \bar{B})$. The sufficient conditions formulated in [15] amount to requiring either that $A$ is not 'too close' to $A_{0}$, or that $B$ is not 'too close' to $B_{0}$, in some appropriate sense. More precisely, they can be expressed as

$$
\lim _{t \rightarrow 0} \sup \left\{\frac{K(t, a ; \bar{A})}{\|a\|_{A}}: 0 \neq a \in A\right\}=0
$$

and

$$
\lim _{t \rightarrow \infty} \inf \left\{\frac{J(t, b ; \bar{B})}{\|b\|_{B}}: 0 \neq b \in B_{0} \cap B_{1}\right\}=\infty
$$

respectively.

There are other very simple conditions that are sufficient for $T: A \rightarrow B$ to be compact. If we also have compactness at the other 'endpoint', i.e. if

$$
T: A_{0} \rightarrow B \text { is compact }
$$

in the first case, or, analogously,

$$
T: A \rightarrow B_{0} \text { is compact }
$$

in the dual case, then it follows immediately that $T: A_{0}+A_{1} \rightarrow B$ is compact, or, in the dual case, that $T: A \rightarrow B_{0} \cap B_{1}$ is compact. This in turn means that $T: A \rightarrow B$ is compact, in the first case, for all interpolation spaces $A$ with respect to $\bar{A}$, and in the second case for all interpolation spaces $B$ with respect to $\bar{B}$.

Remark 1.1. In fact, to obtain the compactness of $T: A \rightarrow B$ when $T$ is compact at both 'endpoints', it is not even necessary to require $A$ or $B$, respectively, to be an interpolation space. A weaker condition, namely $A \hookrightarrow A_{0}+A_{1}$ or $B_{0} \cap B_{1} \hookrightarrow B$, is all that is needed.

In this paper we obtain a quantitative version of the Lions-Peetre Lemma and Theorem 1 of [15], in terms of the measure of non-compactness. We also investigate whether 
it is possible to obtain the results of Lions and Peetre without invoking either of the sufficient conditions discussed above, i.e. what can be said if $A$ is merely an interpolation space with respect to $\bar{A}$ in the first context, or $B$ is merely an interpolation space with respect to $\bar{B}$ in the dual setting? As we show in $\S 3$, these minimal assumptions lead to the conclusion that either $T: A \rightarrow B$ is compact, or a certain relation holds between $A$ and $A_{0}$ or, respectively, between $B$ and $B_{0}$. Furthermore, under mild additional conditions on the couple $\bar{A}$ or $\bar{B}$, respectively, the compactness of $T: A \rightarrow B$ turns out to be equivalent to requiring one or both of the above sufficient conditions (1.1) and (1.3) (or, respectively, (1.2) and (1.4)) to hold. This shows that in some sense these conditions are optimal.

Note that the preceding results are formulated for specific choices of the range space or, respectively, the domain space. But we also show another aspect of the optimality of conditions (1.1) and (1.2) in the context where compactness is required to hold for all possible range spaces or domain spaces, respectively. We prove (again under mild additional assumptions) that, for given $A_{0}, A_{1}$ and $A$, condition (1.1) is necessary and sufficient for $T: A \rightarrow B$ to be compact for all Banach spaces $B$ and all operators $T$ that map $A_{0}$ to $B$ boundedly and $A_{1}$ to $B$ compactly. We obtain a corresponding dual result about the optimality of condition (1.2) without requiring any additional assumptions.

We also show, in $\S 4$, that this sort of behaviour is not an exclusive property of compact operators: similar results hold for operators belonging to any other injective surjective closed operator ideal.

The conditions $A \in \mathcal{C}_{K}(\theta, \bar{A})$ and $B \in \mathcal{C}_{J}(\theta, \bar{B})$ do not in fact stipulate that $A$ is an interpolation space with respect to $\bar{A}$ nor that $B$ is an interpolation space with respect to $\bar{B}$. Furthermore, Remark 1.1 provides a further hint that it is not entirely natural to require $A$ or $B$ to be interpolation spaces in the context of the questions being considered here. It turns out, in fact, that most of our results apply when $A$ and $B$ are in the larger class of interpolation spaces with respect to rank-one operators, or, in some cases, when they are merely intermediate spaces.

\section{Notation and preliminaries}

We recall several standard notions from interpolation theory (cf. [1], [2] and [4]). Let $\bar{A}=\left(A_{0}, A_{1}\right)$ be a Banach couple, that is, two Banach spaces $A_{j}, j=0,1$, which are continuously embedded in some Hausdorff topological vector space. For each $t>0$ we put

$$
J(t, a)=J(t, a ; \bar{A})=\max \left\{\|a\|_{A_{0}}, t\|a\|_{A_{1}}\right\}, \quad \text { for each } a \in A_{0} \cap A_{1},
$$

and

$$
\begin{aligned}
K(t, a) & =K(t, a ; \bar{A}) \\
& =\inf \left\{\left\|a_{0}\right\|_{A_{0}}+t\left\|a_{1}\right\|_{A_{1}}: a=a_{0}+a_{1}, a_{j} \in A_{j}\right\}, \quad \text { for each } a \in A_{0}+A_{1} .
\end{aligned}
$$

Then $\{K(t, \cdot)\}_{t>0}$ is a family of norms on $A_{0}+A_{1}$, any two of which are equivalent. The family $\{J(t, \cdot)\}_{t>0}$ has similar properties on $A_{0} \cap A_{1}$. 
Let $\bar{B}=\left(B_{0}, B_{1}\right)$ be a second Banach couple. Then the notation $T: \bar{A} \rightarrow \bar{B}$ means that $T$ is a linear operator from $A_{0}+A_{1}$ into $B_{0}+B_{1}$, whose restriction to $A_{j}$ defines a bounded operator from $A_{j}$ into $B_{j}$ for $j=0$ and $j=1$. We denote the space of all such operators by $\mathcal{L}(\bar{A}, \bar{B})$, just as $\mathcal{L}(A, B)$ denotes, as usual, the space of all bounded operators mapping a Banach space $A$ into a Banach space $B$.

For each $T \in \mathcal{L}(\bar{A}, \bar{B})$ we introduce the norm

$$
\|T\|_{\bar{A}, \bar{B}}:=\max \left\{\|T\|_{A_{0}, B_{0}},\|T\|_{A_{1}, B_{1}}\right\} .
$$

If one of the couples $\bar{A}$ or $\bar{B}$ reduces to a single Banach space, i.e. if $A_{0}=A_{1}=A$ or if $B_{0}=B_{1}=B$, then we write $T: A \rightarrow \bar{B}$ and $T \in \mathcal{L}(A, \bar{B})$ and denote the norm by $\|T\|_{A, \bar{B}}$ or, respectively, $T: \bar{A} \rightarrow B$ and $T \in \mathcal{L}(\bar{A}, B)$ with norm $\|T\|_{\bar{A}, B}$.

A Banach space $A$ is said to be an intermediate space with respect to $\bar{A}$ if

$$
A_{0} \cap A_{1} \hookrightarrow A \hookrightarrow A_{0}+A_{1},
$$

where, as usual, the notation $\hookrightarrow$ means continuous inclusion. To each intermediate space $A$ it is possible to associate two other intermediate spaces. The first of these, denoted by $A^{\circ}$ and termed the clintersect of $A$, is the closed subspace of $A$ generated by $A_{0} \cap A_{1}$. The second, denoted by $A^{\backsim}$ and termed the Gagliardo completion of $A$, consists of all those $a \in A_{0}+A_{1}$ for which there exists a sequence $\left\{a_{n}\right\}_{n \in \mathbb{N}}$ in some bounded subset of $A$ which converges to $a$ in $A_{0}+A_{1}$. It is normed by an appropriate infimum over all such sequences, i.e.

$$
\|a\|_{A \curvearrowleft}=\inf _{\left\{a_{n}\right\}}\left\{\sup _{n \in \mathbb{N}}\left\{\left\|a_{n}\right\|_{A}\right\}\right\}
$$

(see $[\mathbf{1 0}],[\mathbf{2}]$ or $[\mathbf{1}])$.

An intermediate space $A$ is an interpolation space if, given any $T: \bar{A} \rightarrow \bar{A}$, the restriction of $T$ to $A$ defines a bounded operator from $A$ into itself. It is a classical result of Aronszajn and Gagliardo (see [2, Theorem 2.4.2, p. 28]) that for each such space $A$ there exists a constant $C=C(A, \bar{A})$ such that

$$
\|T\|_{A, A} \leqslant C\|T\|_{\bar{A}, \bar{A}}
$$

for all operators $T: \bar{A} \rightarrow \bar{A}$.

The most familiar examples of interpolation spaces with respect to a given couple $\bar{A}=\left(A_{0}, A_{1}\right)$ are the real interpolation spaces $\left(A_{0}, A_{1}\right)_{\theta, q}$ and the complex interpolation spaces $\left[A_{0}, A_{1}\right]_{\theta}$, which are defined for each $\theta \in(0,1)$ and $q \in[1, \infty]$. Furthermore, $\left(A_{0}, A_{1}\right)_{\theta, q}$ and $\left[A_{0}, A_{1}\right]_{\theta}$ are examples of spaces $A$ that are in both of the classes $\mathcal{C}_{J}(\theta, \bar{A})$ and $\mathcal{C}_{K}(\theta, \bar{A})$. In other words, for some constant $C$ and for all $t>0$ they satisfy each of the two conditions

$$
\|a\|_{A} \leqslant C t^{-\theta} J(t, a), \quad \text { for all } a \in A_{0} \cap A_{1}
$$

and

$$
K(t, a) \leqslant C t^{\theta}\|a\|_{A}, \quad \text { for all } a \in A .
$$


Condition (2.2) is equivalent to the continuous inclusion $\left(A_{0}, A_{1}\right)_{\theta, 1} \hookrightarrow A$ and (2.3) is equivalent to $A \hookrightarrow\left(A_{0}, A_{1}\right)_{\theta, \infty}($ see $[2, \S 3.5])$. However, there are also other interpolation spaces that do not satisfy one or both of these properties for any value of $\theta \in(0,1)$. Obviously, these include the 'endpoint' spaces $A_{0}$ and $A_{1}$ themselves. There are also two other examples of such interpolation spaces which will play special roles in our results here. The first of these is $A_{0}^{\sim}$, the Gagliardo completion of $A_{0}$. It is easy to show (see, for example, [1, Theorem 5.1.4]) that

$$
\|a\|_{A_{0}}=\lim _{t \rightarrow \infty} K(t, a ; \bar{A}) .
$$

(Note that, analogously, the norm of the Gagliardo completion of $A_{1}$ satisfies $\|a\|_{A_{1}}=$ $\lim _{t \rightarrow 0}(1 / t) K(t, a ; \bar{A})$.)

Our second example of such an interpolation space is $A_{0}^{\circ}$, the clintersect of $A_{0}$.

As mentioned in $\S 1$, it turns out to be natural for our purposes here to work with another class of intermediate spaces that includes the interpolation spaces as a subclass. This class is defined with the help of rank-one operators $T: \bar{A} \rightarrow \bar{A}$ of the form $T=f \otimes a$, i.e. $T x=f(x) a$, where $a$ is a fixed element of $A_{0} \cap A_{1}$ and $f$ is a fixed bounded linear functional on $A_{0}+A_{1}$. Obviously, each such $T$ is bounded on $A_{0}$ and $A_{1}$ and on each intermediate space $A$ with respect to $\bar{A}$. But its norms on each of these spaces depend on the particular choices of $a$ and $f$. We shall say that an intermediate space $A$ with respect to the couple $\bar{A}$ is a rank-one interpolation space if, for some constant $C$ depending only on $A$ and $\bar{A}$, it satisfies (2.1) for all $T$ of the special form $T=f \otimes a$.

Rank-one interpolation spaces have been used in a number of papers, $[\mathbf{9}]$ and [19] for example, where they are referred to as 'partly interpolation spaces'.

We shall need to use two functions which in some sense measure the 'position' of a given intermediate space within a Banach couple. These are variants of functions which have been introduced and studied by Dmitriev and by Pustylnik (see [9], [18, pp. 333-334] and $[\mathbf{1 9}$, p. 307]). They are defined as follows.

Definition 2.1. Let $\bar{A}=\left(A_{0}, A_{1}\right)$ be a Banach couple and let $A$ be an intermediate space with respect to $\bar{A}$. For each $t>0$ set

$$
\psi(t)=\psi(t, A, \bar{A}):=\sup \left\{K(t, a):\|a\|_{A}=1\right\}
$$

and

$$
\rho(t)=\rho(t, A, \bar{A}):=\inf \left\{J(t, a): a \in A_{0} \cap A_{1},\|a\|_{A}=1\right\} .
$$

Clearly, $\psi(t)$ and $\rho(t)$ are strictly positive, and they are also both non-decreasing, while $\psi(t) / t$ and $\rho(t) / t$ are non-increasing. We note that these functions are related to the 'embedding functions' $C(\alpha, \beta)$ and $D(\alpha, \beta)$ of $[\mathbf{1 9}$, p. 307] by

$$
C(\alpha, \beta)=\frac{1}{(1 / \alpha) \rho(\alpha / \beta)} \quad \text { and } \quad D(\alpha, \beta)=\frac{1}{(1 / \alpha) \psi(\alpha / \beta)} .
$$

We also note that the conditions (1.1) and (1.2) stated in the introduction correspond to $\lim _{t \rightarrow 0} \psi(t, A, \bar{A})=0$ and $\lim _{t \rightarrow \infty} \rho(t, B, \bar{B})=\infty$, respectively. 
We refer to $[\mathbf{1 9}]$ for some examples indicating the behaviour of the functions $C$ and $D$ in specific cases.

It is possible to characterize rank-one interpolation spaces $A$ by a simple condition on the functions $\psi(t, A, \bar{A})$ and $\rho(t, A, \bar{A})$. This was done by Dmitriev [9] under some additional hypotheses and, in general, by Pustylnik [19]. The following theorem is almost the same as Theorem 1 in $[\mathbf{1 9}$, p. 309].

Theorem 2.2. Let $\bar{A}=\left(A_{0}, A_{1}\right)$ be a Banach couple and let $A$ be an intermediate space with respect to $\bar{A}$. Then $A$ is a rank-one interpolation space if and only if there exists a constant $C$ depending only on $A$ and $\bar{A}$ such that

$$
\psi(t, A, \bar{A}) \leqslant C \rho(t, A, \bar{A}), \quad \text { for all } t>0 .
$$

Proof. In view of (2.4), the proof is a very slight and obvious modification of the proof given in $[\mathbf{1 9}]$.

\section{Compactness results}

As pointed out by Mastyło, the proof of the generalized Lions-Peetre compactness Lemma, assuming the weaker conditions (1.1) and (1.2) instead of $A \in \mathcal{C}_{K}(\theta, \bar{A})$ and $B \in \mathcal{C}_{J}(\theta, \bar{B})$, is essentially the same as the proof of the original lemma (cf. [2, pp. 56 $57])$.

Here, however, we shall adopt a quantitative approach. The following two theorems describe interpolation properties of the measure of non-compactness. They can be applied in fact to any intermediate space and imply the generalized compactness lemma. Their estimates also point the way to results to be presented in $\S 4$.

Let us recall that the (ball) measure of non-compactness $\beta(T)=\beta\left(T_{A, B}\right)$ of an operator $T \in \mathcal{L}(A, B)$ is defined to be the infimum of the set of all numbers $\sigma>0$ for which there exists a finite subset $B(\sigma) \subset B$ such that

$$
T\left(U_{A}\right) \subset \bigcup_{b \in B(\sigma)}\left(b+\sigma U_{B}\right) .
$$

Here, $U_{A}$ (respectively, $U_{B}$ ) denotes the closed unit ball of $A$ (respectively, $B$ ). Of course, $T$ is compact if and only if $\beta(T)=0$.

Theorem 3.1. Let $\bar{A}=\left(A_{0}, A_{1}\right)$ be a Banach couple, let $A$ be an intermediate space with respect to $\bar{A}$ and let $B$ be another Banach space. Then, for each $T \in \mathcal{L}(\bar{A}, B)$ :

(a) if $\beta\left(T_{A_{0}, B}\right)=0$, then

$$
\beta\left(T_{A, B}\right) \leqslant \beta\left(T_{A_{1}, B}\right) \cdot \lim _{t \rightarrow \infty} \frac{\psi(t, A, \bar{A})}{t} ;
$$

(b) if $\beta\left(T_{A_{1}, B}\right)=0$, then

$$
\beta\left(T_{A, B}\right) \leqslant \beta\left(T_{A_{0}, B}\right) \cdot \lim _{t \rightarrow 0} \psi(t, A, \bar{A})
$$

and 
(c) if $\beta\left(T_{A_{i}, B}\right)>0$ for $i=0,1$, then

$$
\beta\left(T_{A, B}\right) \leqslant 2 \beta\left(T_{A_{0}, B}\right) \psi\left(\frac{\beta\left(T_{A_{1}, B}\right)}{\beta\left(T_{A_{0}, B}\right)}, A, \bar{A}\right) .
$$

Proof. For each $\epsilon>0$ and for $i=0,1$ there exists a finite set $B(\epsilon, i) \subset B$ such that

$$
T\left(U_{A_{i}}\right) \subset \bigcup_{b \in B(\epsilon, i)}\left(b+\left(\epsilon+\beta\left(T_{A_{i}, B}\right)\right) U_{B}\right) .
$$

Now, consider an arbitrary element $a \in U_{A}$ and fixed positive numbers $t$ and $\delta$. Since $K(t, a)<\delta+\psi(t)$, there exists a decomposition $a=a_{0}+a_{1}$ with $a_{i} \in A_{i}$ for $i=0,1$ such that $\left\|a_{0}\right\|_{A_{0}}+t\left\|a_{1}\right\|_{A_{1}}<\delta+\psi(t)$. Thus,

$$
a_{i} \in t^{-i}(\delta+\psi(t)) U_{A_{i}}, \quad \text { for } i=0,1 .
$$

Consequently, by (3.1), for some $b_{i} \in B(\epsilon, i)$, we have

$$
T a_{i} \in t^{-i}(\delta+\psi(t)) b_{i}+t^{-i}(\delta+\psi(t))\left(\epsilon+\beta\left(T_{A_{i}, B}\right)\right) U_{B}, \quad \text { for } i=0,1,
$$

which implies that

$$
T a \in(\delta+\psi(t))\left(b_{0}+\frac{1}{t} b_{1}\right)+(\delta+\psi(t))\left(\epsilon+\beta\left(T_{A_{0}, B}\right)+\frac{1}{t}\left(\epsilon+\beta\left(T_{A_{1}, B}\right)\right)\right) U_{B} .
$$

Since the subset of $B$ consisting of all elements of the form $(\delta+\psi(t))\left(b_{0}+(1 / t) b_{1}\right)$ for some $b_{0} \in B(\epsilon, 0)$ and $b_{1} \in B(\epsilon, 1)$ is of course finite, we have shown that

$$
\beta\left(T_{A, B}\right) \leqslant(\delta+\psi(t))\left(\epsilon+\beta\left(T_{A_{0}, B}\right)+\frac{1}{t}\left(\epsilon+\beta\left(T_{A_{1}, B}\right)\right)\right) .
$$

This in turn implies, since $\epsilon$ and $\delta$ can both be chosen arbitrarily small, that

$$
\beta\left(T_{A, B}\right) \leqslant \psi(t)\left(\beta\left(T_{A_{0}, B}\right)+\frac{1}{t} \beta\left(T_{A_{1}, B}\right)\right), \quad \text { for all } t>0 .
$$

We can now immediately obtain cases (a) and (b) of the theorem by substituting $\beta\left(T_{A_{i}, B}\right)=0$ in (3.2) for $i=0$ or 1 , and using the facts that $\psi(t)$ is non-decreasing and $\psi(t) / t$ is non-increasing. Case (c) also follows from (3.2) by simply substituting $t=\beta\left(T_{A_{1}, B}\right) / \beta\left(T_{A_{0}, B}\right)$.

Now we turn to the analogous theorem for the 'dual' situation.

Theorem 3.2. Let $\bar{B}=\left(B_{0}, B_{1}\right)$ be a Banach couple, let $B$ be an intermediate space with respect to $\bar{B}$ and let $A$ be another Banach space. Then, for each operator $T \in$ $\mathcal{L}(A, \bar{B})$ :

(a) if $\beta\left(T_{A, B_{0}}\right)=0$, then

$$
\beta\left(T_{A, B}\right) \leqslant 2 \beta\left(T_{A, B_{1}}\right) \cdot \lim _{t \rightarrow 0} \frac{t}{\rho(t, B, \bar{B})} ;
$$


(b) if $\beta\left(T_{A, B_{1}}\right)=0$, then

$$
\beta\left(T_{A, B}\right) \leqslant 2 \beta\left(T_{A, B_{0}}\right) \cdot \lim _{t \rightarrow \infty} \frac{1}{\rho(t, B, \bar{B})} ;
$$

and

(c) if $\beta\left(T_{A, B_{i}}\right)>0$ for $i=0,1$, then

$$
\beta\left(T_{A, B}\right) \leqslant \frac{2 \beta\left(T_{A, B_{0}}\right)}{\rho\left(\beta\left(T_{A, B_{0}}\right) / \beta\left(T_{A, B_{1}}\right), B, \bar{B}\right)} .
$$

Proof. For each fixed $\epsilon>0$ there exist finite subsets $B(\epsilon, 0)$ of $B_{0}$ and $B(\epsilon, 1)$ of $B_{1}$ such that

$$
T\left(U_{A}\right) \subset \bigcup_{b \in B(\epsilon, i)}\left(b+\left(\epsilon+\beta\left(T_{A, B_{i}}\right)\right) U_{B_{i}}\right), \quad \text { for } i=0,1
$$

Let us now construct a new set $\tilde{B}(\epsilon)$ of elements of $B_{0} \cap B_{1}$ in the following way: For each choice of $b_{0} \in B(\epsilon, 0)$ and $b_{1} \in B(\epsilon, 1)$, if the set $E\left(b_{0}, b_{1}\right)=\left(b_{0}+\left(\epsilon+\beta\left(T_{A, B_{0}}\right)\right) U_{B_{0}}\right) \cap$ $\left(b_{1}+\left(\epsilon+\beta\left(T_{A, B_{1}}\right)\right) U_{B_{1}}\right)$ is non-empty, choose exactly one element $w\left(b_{0}, b_{1}\right)$ from this set, i.e. we set

$$
\tilde{B}(\epsilon)=\left\{w\left(b_{0}, b_{1}\right): b_{0} \in B(\epsilon, 0), b_{1} \in B(\epsilon, 1), E\left(b_{0}, b_{1}\right) \neq \emptyset\right\} .
$$

Clearly, $\tilde{B}(\epsilon)$ is a non-empty finite set.

Given an arbitrary fixed element $a \in U_{A}$, it follows from (3.3) that there exist $b_{0} \in$ $B(\epsilon, 0)$ and $b_{1} \in B(\epsilon, 1)$ such that $T a \in\left(b_{0}+\left(\epsilon+\beta\left(T_{A, B_{0}}\right)\right) U_{B_{0}}\right) \cap\left(b_{1}+\left(\epsilon+\beta\left(T_{A, B_{1}}\right)\right) U_{B_{1}}\right)$. Hence, $E\left(b_{0}, b_{1}\right) \neq \emptyset$, and so the element $w=w\left(b_{0}, b_{1}\right) \in \tilde{B}(\epsilon)$ must satisfy

$$
\|T a-w\|_{B_{0}} \leqslant\left\|T a-b_{0}\right\|_{B_{0}}+\left\|b_{0}-w\right\|_{B_{0}} \leqslant 2\left(\epsilon+\beta\left(T_{A, B_{0}}\right)\right),
$$

and also

$$
\|T a-w\|_{B_{1}} \leqslant\left\|T a-b_{1}\right\|_{B_{1}}+\left\|b_{1}-w\right\|_{B_{1}} \leqslant 2\left(\epsilon+\beta\left(T_{A, B_{1}}\right)\right) .
$$

Consequently, for each $t>0$,

$$
\begin{aligned}
\|T a-w\|_{B} & \leqslant \frac{1}{\rho(t, B, \bar{B})} J(t, T a-w ; \bar{B}) \\
& \leqslant \frac{2}{\rho(t, B, \bar{B})} \max \left\{\epsilon+\beta\left(T_{A, B_{0}}\right), t\left(\epsilon+\beta\left(T_{A, B_{1}}\right)\right)\right\} .
\end{aligned}
$$

It follows that

$$
\beta\left(T_{A, B}\right) \leqslant \frac{2}{\rho(t, B, \bar{B})} \max \left\{\epsilon+\beta\left(T_{A, B_{0}}\right), t\left(\epsilon+\beta\left(T_{A, B_{1}}\right)\right)\right\},
$$

and so, since $\epsilon$ is arbitrary, we obtain

$$
\beta\left(T_{A, B}\right) \leqslant \frac{2}{\rho(t, B, \bar{B})} \max \left\{\beta\left(T_{A, B_{0}}\right), t \beta\left(T_{A, B_{1}}\right)\right\} .
$$


To deal with cases (a) and (b) of the theorem, we simply substitute $\beta\left(T_{A, B_{i}}\right)=0$ for $i=0$ or $i=1$ in (3.4) and use the facts that $1 / \rho(t)$ is non-increasing and $t / \rho(t)$ is non-decreasing. We obtain case (c) by substituting $t=\beta\left(T_{A, B_{0}}\right) / \beta\left(T_{A, B_{1}}\right)$.

Now that we have prepared a number of tools, we can begin looking more closely at various ways in which natural generalizations of the Lions-Peetre compactness results may fail to hold.

Let us first consider the setting where we have $T: \bar{A} \rightarrow B$ and $T: A_{1} \rightarrow B$ is compact. If $T: A_{0} \rightarrow B$ is not compact, then there may exist intermediate spaces or even interpolation spaces $A$ such that $T: A \rightarrow B$ is not compact. One trivial example is to take $A=A_{0}$. But the following example is more relevant to the general result that we shall obtain.

Example 3.3. Let $\bar{A}=\left(A_{0}, A_{1}\right)=\left(\ell_{\infty}, \ell_{\infty}\left(2^{n}\right)\right)$, where $\ell_{\infty}$ is the usual space of scalar sequences $\xi=\left\{\xi_{n}\right\}_{n \in \mathbb{N}}$ satisfying $\|\xi\|_{\ell_{\infty}}:=\sup _{n \in \mathbb{N}}\left|\xi_{n}\right|<\infty$ and $\ell_{\infty}\left(2^{n}\right)$ is the corresponding 'weighted' space of those sequences $\xi$ for which $\|\xi\|_{\ell_{\infty}\left(2^{n}\right)}:=\sup _{n \in \mathbb{N}}\left|2^{n} \xi_{n}\right|<\infty$. We choose $A=c_{0}$, the subspace of sequences in $\ell_{\infty}$ that converge to zero. This is an interpolation space since $A=A_{0}^{\circ}$. Then we take $B=\ell_{\infty}$ and let $T$ be the identity operator $T \xi=\xi$. Clearly, $T: \bar{A} \rightarrow B$ and also $T: A_{1} \rightarrow B$ is compact. Nevertheless, $T: A \rightarrow B$ is the inclusion from $c_{0}$ into $\ell_{\infty}$ which is not compact.

Remark 3.4. Note that whenever we have, as in the preceding example, that

$$
A_{0}^{\circ} \hookrightarrow A,
$$

and also that $T: A_{0}^{\circ} \rightarrow B$ is not compact, then of course $T: A \rightarrow B$ cannot be compact.

Let us now consider the 'dual' situation, where $T: A \rightarrow \bar{B}$ and $T: A \rightarrow B_{1}$ is compact. Analogously to before, if $T: A \rightarrow B_{0}$ is not compact, then $T: A \rightarrow B$ will not be compact in general for all intermediate spaces or even interpolation spaces $B$. Again, in addition to the obvious example $B=B_{0}$, we have another example that is more relevant for the results that we shall soon formulate.

Example 3.5. Let

$$
\bar{B}=\left(B_{0}, B_{1}\right)=\left(c_{0}, \ell_{\infty}\left(2^{-n}\right)\right), \quad \text { where }\|\xi\|_{\ell_{\infty}\left(2^{-n}\right)}:=\sup _{n \in \mathbb{N}}\left|2^{-n} \xi_{n}\right|,
$$

and let $B=\ell_{\infty}$. It is easy to see that $\ell_{\infty}$ is the Gagliardo completion of $B_{0}=c_{0}$ in $B_{0}+B_{1}=\ell_{\infty}\left(2^{-n}\right)$. Thus, $B$ is an interpolation space with respect to $\bar{B}$. We now choose $A=c_{0}$ and again let $T$ be the identity operator. Then $T: A \rightarrow \bar{B}$ and $T: A \rightarrow B_{1}$ is compact, but $T: A \rightarrow B$ is not compact because (as in the previous example) it is the inclusion from $c_{0}$ into $\ell_{\infty}$.

Remark 3.6. The condition analogous to (3.5) in this case is

$$
B \hookrightarrow B_{0}^{\backsim} .
$$

Whenever it holds (as it does of course in Example 3.5) and $T: A \rightarrow B_{0}^{\backsim}$ is not compact, then $T: A \rightarrow B$ cannot be compact. We shall soon see, in fact, that the only mechanisms 
that can prevent a compactness result of Lions-Peetre type from holding for an arbitrary interpolation or rank-one interpolation space are those described in this remark and in Remark 3.4.

The next two lemmas will sometimes enable us to deduce that the conditions mentioned in Remarks 3.4 and 3.6 hold when either $A$ or $B$, respectively, is a rank-one interpolation space.

Lemma 3.7. Let $\bar{A}=\left(A_{0}, A_{1}\right)$ be a Banach couple and let $A$ be a rank-one interpolation space with respect to $\bar{A}$.

(i) If $\lim _{t \rightarrow 0} \psi(t, A, \bar{A})>0$, then $A_{0}^{\circ} \hookrightarrow A$.

(ii) If $\lim _{t \rightarrow \infty}(\psi(t, A, \bar{A}) / t)>0$, then $A_{1}^{\circ} \hookrightarrow A$.

Proof. Suppose $\lim _{t \rightarrow 0} \psi(t)>0$. Then, by Theorem 2.2, we have $\lim _{t \rightarrow 0} \rho(t, A, \bar{A})=$ $M>0$. Since $\rho$ is non-decreasing, it follows that $M \leqslant\left(J(t, a) /\|a\|_{A}\right)$ for all $t>0$ and all $a \in A_{0} \cap A_{1}$. Therefore, $\|a\|_{A} \leqslant(1 / M) \max \left\{\|a\|_{A_{0}}, t\|a\|_{A_{1}}\right\}$. Since we may choose $t=\|a\|_{A_{0}} /\|a\|_{A_{1}}$, this implies that $\|a\|_{A} \leqslant(1 / M)\|a\|_{A_{0}}$ for all $a \in A_{0} \cap A_{1}$, and, consequently, $A_{0}^{\circ} \hookrightarrow A$.

The proof of (ii) is similar.

Lemma 3.8. Let $\bar{B}=\left(B_{0}, B_{1}\right)$ be a Banach couple and let $B$ be a rank-one interpolation space with respect to $\bar{B}$.

(i) If $\lim _{t \rightarrow \infty}(1 / \rho(t, B, \bar{B}))>0$, then $B \hookrightarrow B_{0}^{\curvearrowleft}$.

(ii) If $\lim _{t \rightarrow 0}(t / \rho(t, B, \bar{B}))>0$, then $B \hookrightarrow B_{1}^{\backsim}$.

Proof. Assume that $\lim _{t \rightarrow \infty}(1 / \rho(t))>0$. Then, by Theorem 2.2, we have that $\lim _{t \rightarrow \infty} \psi(t, B, \bar{B})=M<\infty$. Consequently, each $b \in B$ satisfies

$$
\|b\|_{B_{0}^{\varkappa}}=\lim _{t \rightarrow \infty} K(t, b ; \bar{B}) \leqslant \lim _{t \rightarrow \infty} \psi(t)\|b\|_{B}=M\|b\|_{B},
$$

and so $B \hookrightarrow B_{0}^{\curvearrowleft}$.

Case (ii) can be treated analogously.

We can now give a precise formulation of our claim made at the end of Remark 3.6. This is done in the following two theorems, which are almost immediate consequences of the preceding results.

Theorem 3.9. Let $\bar{A}=\left(A_{0}, A_{1}\right)$ be a Banach couple, let $A$ be a rank-one interpolation space with respect to $\bar{A}$ and let $B$ be another Banach space. Let $T: \bar{A} \rightarrow B$ be a linear operator such that $T: A_{1} \rightarrow B$ is compact. Then at least one of the following conditions must hold.

(i) $T: A \rightarrow B$ is compact.

(ii) $A_{0}^{\circ} \hookrightarrow A$. 
Proof. Since $\beta\left(T_{A_{1}, B}\right)=0$, Theorem 3.1 implies that

$$
\beta\left(T_{A, B}\right) \leqslant \beta\left(T_{A_{0}, B}\right) \cdot \lim _{t \rightarrow 0} \psi(t, A, \bar{A}) .
$$

Consequently, either $T: A \rightarrow B$ is compact, i.e. $\beta\left(T_{A, B}\right)=0$, or, alternatively, we have $\beta\left(T_{A, B}\right)>0$. In this latter case we must have $\lim _{t \rightarrow 0} \psi(t, A, \bar{A})>0$, which, by Lemma 3.7, implies that $A_{0}^{\circ} \hookrightarrow A$.

Here is the analogous result for the 'dual' situation.

Theorem 3.10. Let $\bar{B}=\left(B_{0}, B_{1}\right)$ be a Banach couple, let $B$ be a rank-one interpolation space with respect to $\bar{B}$ and let $A$ be another Banach space. Let $T: A \rightarrow \bar{B}$ be a linear operator such that $T: A \rightarrow B_{1}$ is compact. Then at least one of the following conditions must hold.

(i) $T: A \rightarrow B$ is compact.

(ii) $B \hookrightarrow B_{0}^{\sim}$.

Proof. According to Theorem 3.2 we have

$$
\beta\left(T_{A, B}\right) \leqslant 2 \beta\left(T_{A, B_{0}}\right) \cdot \lim _{t \rightarrow \infty} \frac{1}{\rho(t, B, \bar{B})} .
$$

So, if $T: A \rightarrow B$ is not compact, we must necessarily have

$$
0<\beta\left(T_{A, B}\right) \leqslant 2 \beta\left(T_{A, B_{0}}\right) \cdot \lim _{t \rightarrow \infty} \frac{1}{\rho(t, B, \bar{B})},
$$

and, consequently, by Lemma $3.8, B \hookrightarrow B_{0}^{\sim}$.

Now, it is easy to show that, under mild additional conditions on the Banach couples, the known sufficient conditions for $T: A \rightarrow B$ to be compact are also necessary.

Corollary 3.11. Let $\bar{A}=\left(A_{0}, A_{1}\right)$ be a Banach couple such that $A_{0}^{\circ}=A_{0}$, let $A$ be a rank-one interpolation space with respect to $\bar{A}$ and let $B$ be another Banach space. Let $T: \bar{A} \rightarrow B$ be a linear operator such that $T: A_{1} \rightarrow B$ is compact. Then $T: A \rightarrow B$ is compact if and only if at least one of the following conditions hold.

(i) $\lim _{t \rightarrow 0} \psi(t, A, \bar{A})=0$.

(ii) $T: A_{0} \rightarrow B$ is compact.

Proof. From Theorem 3.1 (b) it is clear that either of conditions (i) and (ii) is sufficient to ensure that $T: A \rightarrow B$ is compact. Conversely, if $T: A \rightarrow B$ is compact and if (i) does not hold, then we deduce from Lemma 3.7 that $A_{0}^{\circ} \hookrightarrow A$, which in turn implies that $T: A_{0}^{\circ} \rightarrow B$ is compact. Since $A_{0}^{\circ}=A_{0}$ we obtain (ii), completing the proof.

Corollary 3.12. Let $\bar{B}=\left(B_{0}, B_{1}\right)$ be a Banach couple such that $B_{0}^{\sim}=B_{0}$, let $B$ be a rank-one interpolation space with respect to $\bar{B}$ and let $A$ be another Banach space. Let $T: A \rightarrow \bar{B}$ be a linear operator such that $T: A \rightarrow B_{1}$ is compact. Then $T: A \rightarrow B$ is compact if and only if at least one of the following conditions hold. 
(i) $\lim _{t \rightarrow \infty} \rho(t, B, \bar{B})=\infty$.

(ii) $T: A \rightarrow B_{0}$ is compact.

Proof. The argument is analogous to the proof of the preceding corollary. By Theorem 3.2 (b) each of conditions (i) and (ii) is sufficient for the compactness of $T: A \rightarrow B$. Conversely, if $T: A \rightarrow B$ is compact and (i) does not hold, then Lemma 3.8 gives that $B \hookrightarrow B_{0}^{\backsim}=B_{0}$, which implies (ii).

Remark 3.13. It is easy to see that the last two corollaries cannot be sharpened to hold without the condition $A_{0}^{\circ}=A_{0}$ or $B_{0}^{\backsim}=B_{0}$, respectively. For example, suppose, in the context of Corollary 3.11, that $A_{1} \hookrightarrow A_{0}$ and that $A$ is also an interpolation space with respect to the couple $\left(A_{0}^{\circ}, A_{1}\right)$. Then the behaviour of $T$ on the space $A$ is completely determined by the behaviour of $T$ on $A_{0}^{\circ}$, and it is completely irrelevant to ask whether or not $T$ maps the larger space $A_{0}$ compactly into $B$. The following example can illustrate this more explicitly. Suppose that $\bar{E}=\left(E_{0}, E_{1}\right)$ is a Banach couple such that $E_{1} \hookrightarrow E_{0}=E_{0}^{\circ}$. Define a new Banach couple $\bar{A}=\left(A_{0}, A_{1}\right)$ by setting $A_{0}=E_{0} \oplus Y$ and $A_{1}=E_{1} \oplus\{0\}$, where $Y$ is an infinite-dimensional Banach space and $\{0\}$ denotes its trivial subspace. (The norms for $A_{0}$ and $A_{1}$ may be taken, say, to be $\|(x, y)\|_{A_{0}}=\max \left\{\|x\|_{E_{0}},\|y\|_{Y}\right\}$ and $\|(x, 0)\|_{A_{1}}=\|x\|_{E_{1}}$.) Each operator $S: \bar{A} \rightarrow \bar{A}$ can be decomposed into a $2 \times 2$ 'matrix'

$$
S=\left(\begin{array}{ll}
S_{11} & S_{12} \\
S_{21} & S_{22}
\end{array}\right),
$$

i.e. $S(x, y)=\left(S_{11} x+S_{12} y, S_{21} x+S_{22} y\right)$ for all $(x, y) \in E_{0} \oplus Y$. Since $S: A_{0} \rightarrow A_{0}$ is bounded, it follows that each of the four operators $S_{11}: E_{0} \rightarrow E_{0}, S_{12}: Y \rightarrow E_{0}$, $S_{21}: E_{0} \rightarrow Y$ and $S_{22}: Y \rightarrow Y$ is bounded. Since $S: A_{1} \rightarrow A_{1}$ is also bounded, we deduce that $S_{11}: E_{1} \rightarrow E_{1}$, i.e. that $S_{11}: \bar{E} \rightarrow \bar{E}$, and also that $S_{21}=0$. From this description of the operator $S$ it follows that whenever $E$ is an interpolation space with respect to $\bar{E}$, then $A=E \oplus\{0\}$ is an interpolation space with respect to $\bar{A}$.

Now, let $B$ be another infinite-dimensional Banach space and consider a bounded operator $T: A_{0} \rightarrow B$ such that $T: A_{1} \rightarrow B$ is compact. This means that $T$ has the form $T(x, y)=T_{1} x+T_{2} y$ for all $x \in E_{0}$ and $y \in Y$, where $T_{1}: E_{0} \rightarrow B$ is bounded, $T_{1}: E_{1} \rightarrow B$ is compact, and $T_{2}: Y \rightarrow B$ is bounded. If $A$ is an interpolation space of the form $A=E \oplus\{0\}$, then $T: A \rightarrow B$ is compact if and only if $T_{1}: E \rightarrow B$ is compact. Consequently, we can apply Corollary 3.11 to the operator $T_{1}: \bar{E} \rightarrow B$. We obtain that $T: A \rightarrow B$ is compact if and only if either $\lim _{t \rightarrow 0} \psi(t, E, \bar{E})=0$ or $T_{1}: E_{0} \rightarrow B$ is compact or both of these conditions hold. The latter condition is the same as the compactness of $T: A_{0}^{\circ} \rightarrow B$, since $A_{0}^{\circ}=E_{0} \oplus\{0\}$. It is now quite clear that in this case the compactness of $T: A_{0} \rightarrow B$ is irrelevant: the compactness of $T: A_{0} \rightarrow B$ is equivalent to the condition that both of the operators $T_{1}: E_{0} \rightarrow B$ and $T_{2}: Y \rightarrow B$ are compact, but the behaviour of $T_{2}$ has no effect on the compactness of $T: A \rightarrow B$.

In the last two results of this section we do not have to confine ourselves to rankone interpolation spaces. Instead we can deal with all intermediate spaces. But, before 
stating those results, we give the details of an example, kindly pointed out to us by Evgeniy Pustylnik, which shows that there is necessarily a difference between the case of rank-one interpolation spaces and general intermediate spaces. More specifically, it shows that we cannot obtain a result analogous to Theorem 3.9 if we assume that $A$ is merely an intermediate space rather than a rank-one interpolation space.

Example 3.14. Let $\bar{A}=\left(A_{0}, A_{1}\right)=\left(L_{p}(0,1), L_{q}(0,1)\right)$, where $1<p<q<\infty$. Let $A$ be the Lorentz space $L_{p, 1}(0,1)$, which is an intermediate space with respect to $\bar{A}$ but not a rank-one space. (This latter claim can also be deduced from what is to follow.) Set

$$
\lambda=1+\frac{1}{q}-\frac{1}{p}
$$

and consider the potential operator $T$ defined by

$$
T x(t)=\int_{0}^{1} \frac{x(s)}{|t-s|^{\lambda}} \mathrm{d} s .
$$

As was shown in [11], $T: L_{p} \rightarrow L_{q}$ boundedly, and, consequently, we also have $T$ : $L_{q} \rightarrow L_{q}$ boundedly. Moreover, by [13, Theorem 2.8.1], $T: L_{q} \rightarrow L_{q}$ is also compact. Despite these properties of $T$, neither of conditions (i) and (ii) in Theorem 3.9 holds for this example. First, we have $A_{0}^{\circ}=A_{0}=L_{p}$, which is not contained in $A=L_{p, 1}$. Furthermore, the sequence

$$
x_{k}(t)=\left\{\begin{array}{ll}
k^{1 / p}, & \text { if } 0<t<(1 / k), \\
0, & \text { if } t \geqslant(1 / k),
\end{array} \quad \text { for all } k \in \mathbb{N}\right.
$$

is bounded in $L_{p, 1}$ because

$$
\left\|x_{k}\right\|_{L_{p, 1}}=\int_{0}^{1} t^{(1 / p)-1} x_{k}^{*}(t) \mathrm{d} t=k^{1 / p} \int_{0}^{1 / k} t^{(1 / p)-1} \mathrm{~d} t=p
$$

but, for any $t \in(0,1 / k)$, we have

$$
\begin{aligned}
T x_{k}(t)=k^{1 / p} \int_{0}^{1 / k} \frac{\mathrm{d} s}{|t-s|^{\lambda}} & \geqslant\left(\frac{1}{p}-\frac{1}{q}\right)^{-1} k^{(1 / p)+\lambda-1} \\
& =\left(\frac{1}{p}-\frac{1}{q}\right)^{-1} k^{1 / q}
\end{aligned}
$$

and, thus,

$$
\left\|T x_{k} \chi_{(0,1 / k)}\right\|_{L_{q}} \geqslant 1, \quad \text { for all } k \in \mathbb{N} .
$$

This means that the functions $T x_{k}$ do not possess equi-absolutely continuous norms in $L_{q}$ as $k \rightarrow \infty$. As was shown in [13], such a sequence of functions is not compact in $L_{q}$. Thus, $T: L_{p, 1} \rightarrow L_{q}$ is not compact.

Our first result for the case of general intermediate spaces is an analogue of Corollary 3.11 , where we simultaneously consider all possible range spaces $B$. 
Theorem 3.15. Let $\bar{A}=\left(A_{0}, A_{1}\right)$ be a Banach couple and let $A$ be an intermediate space with respect to $\bar{A}$. Suppose that either $A \cap A_{1}$ is dense in $A$, or that $A_{0} \cap A_{1}$ is dense in $A_{0}$, or that

$$
\lim _{t \rightarrow 0} K(t, a ; \bar{A})=0, \quad \text { for all } a \in A \text {. }
$$

Then the following are equivalent.

(i) $\lim _{t \rightarrow 0} \psi(t, A, \bar{A})=0$.

(ii) For every Banach space $B$, if $T: \bar{A} \rightarrow B$ is a linear operator such that $T: A_{1} \rightarrow B$ is compact, then $T: A \rightarrow B$ is compact.

(iii) If $T: \bar{A} \rightarrow \ell_{\infty}$ is a linear operator such that $T: A_{1} \rightarrow \ell_{\infty}$ is compact, then $T: A \rightarrow \ell_{\infty}$ is compact.

Remark 3.16. Since every separable Banach space can be isometrically embedded into $\ell_{\infty}$, it is not surprising that it is sufficient to check condition (ii) just for the special case $B=\ell_{\infty}$. It will be clear from the proof that if $A_{0}^{\circ}=A_{0}$, then it is also sufficient to check for the special case $B=c_{0}$.

Proof. Let us first observe that the condition that we really need here is (3.7). It is easy to verify that it is a consequence of either of the two density conditions in the statement of the theorem.

Once again we use Theorem 3.1 (b) to obtain that (i) implies (ii). Of course (ii) implies (iii). (Both of these implications also hold when the additional density conditions or (3.7) are not satisfied.) To complete the proof we will show that (iii) implies (i), or, equivalently, that if (i) does not hold, then (iii) cannot hold.

Thus we assume that $\lim _{t \rightarrow 0} \psi(t, A, \bar{A})=\delta>0$, which implies that there exist a sequence $\left\{a_{n}\right\}_{n \in \mathbb{N}}$ of elements in $U_{A}$ and a decreasing sequence $\left\{t_{n}\right\}_{n \in \mathbb{N}}$ of numbers in $(0,1)$ such that $\lim _{n \rightarrow \infty} t_{n}=0$ and $K\left(t_{n}, a_{n} ; \bar{A}\right)>\delta / 2$. In view of (3.7) we can also assume, by passing if necessary to subsequences of the original sequences, that

$$
K\left(t_{n}, a_{m} ; \bar{A}\right)<\delta / 4, \quad \text { for all } m<n .
$$

By the Hahn-Banach theorem, for each $n \in \mathbb{N}$, there exists a linear functional $f_{n}$ on $A_{0}+A_{1}$ such that $f_{n}\left(a_{n}\right)=K\left(t_{n}, a_{n} ; \bar{A}\right)$ and $\left|f_{n}(a)\right| \leqslant K\left(t_{n}, a ; \bar{A}\right)$ for each $a \in A_{0}+A_{1}$. Now consider the linear operator $T: A_{0}+A_{1} \rightarrow \ell_{\infty}$ defined by $T a=\left\{f_{n}(a)\right\}_{n \in \mathbb{N}}$. Clearly, $T: A_{i} \rightarrow \ell_{\infty}$ with norm not exceeding 1 for $i=0,1$. Furthermore, $T$ maps the unit ball of $A_{1}$ into the set of all sequences $\left\{\lambda_{n}\right\}_{n \in \mathbb{N}}$ that satisfy $\left|\lambda_{n}\right| \leqslant t_{n}$. This is clearly a compact subset of $c_{0}$ and of $\ell_{\infty}$. On the other hand, for each pair of positive integers $m<n$ we have

$$
\left\|T a_{n}-T a_{m}\right\|_{\ell_{\infty}} \geqslant\left|f_{n}\left(a_{n}\right)-f_{n}\left(a_{m}\right)\right| \geqslant K\left(t_{n}, a_{n}\right)-K\left(t_{n}, a_{m}\right)>\frac{1}{4} \delta .
$$

Thus, $T: A \rightarrow \ell_{\infty}$ is not compact, and so (iii) does not hold. 
Finally we present a result analogous to Corollary 3.12 which is essentially a dual of the preceding theorem. This time we simultaneously consider all possible domain spaces $A$. In contrast to the previous theorem, we do not need to impose any additional conditions.

Theorem 3.17. Let $\bar{B}=\left(B_{0}, B_{1}\right)$ be a Banach couple and let $B$ be an intermediate space with respect to $\bar{B}$. Then the following are equivalent.

(i) $\lim _{t \rightarrow \infty} \rho(t, B, \bar{B})=\infty$.

(ii) For every Banach space $A$, if $T: A \rightarrow \bar{B}$ is a linear operator such that $T: A \rightarrow B_{1}$ is compact, then $T: A \rightarrow B$ is compact.

(iii) If $T: \ell_{1} \rightarrow \bar{B}$ is a linear operator such that $T: \ell_{1} \rightarrow B_{1}$ is compact, then $T: \ell_{1} \rightarrow B$ is compact.

Proof. We obtain (i) $\Rightarrow$ (ii) by Theorem 3.2 (b). Obviously, (ii) $\Rightarrow$ (iii). To show that (iii) $\Rightarrow(\mathrm{i})$, let us suppose that (i) does not hold. Then $\lim _{t \rightarrow \infty} \rho(t, B, \bar{B})=\delta \in$ $(0, \infty)$ and there exist a sequence $\left\{b_{n}\right\}_{n \in \mathbb{N}}$ of elements in $B_{0} \cap B_{1}$ with $\left\|b_{n}\right\|_{B}=1$ and $\left\|b_{n}\right\|_{B_{0}}<2 \delta$ and a sequence $\left\{t_{n}\right\}_{n \in \mathbb{N}}$ of numbers in $(1, \infty)$ such that $\lim _{n \rightarrow \infty} t_{n}=\infty$ and $\left\|b_{n}\right\|_{B_{1}}<2 \delta / t_{n}$. We consider the operator $T: \ell_{1} \rightarrow B_{0}+B_{1}$ defined by

$$
T\left(\left\{\lambda_{n}\right\}_{n \in \mathbb{N}}\right)=\sum_{n \in \mathbb{N}} \lambda_{n} b_{n} .
$$

Clearly, $T: \ell_{1} \rightarrow B_{i}$ for $i=0,1$ with norm not exceeding $2 \delta$. Furthermore, $T: \ell_{1} \rightarrow B_{1}$ is compact, as the limit, in operator norm, of a sequence of finite-rank operators. However, $T: \ell_{1} \rightarrow B$ is not compact. This follows immediately from the fact that the sequence $\left\{b_{n}\right\}$ does not contain any subsequence that converges in $B$. Were $\left\{b_{m_{n}}\right\}$ to be such a sequence, its limit $b_{*}$ would necessarily satisfy $\left\|b_{*}\right\|_{B}=1$. But this would be a contradiction, since this same sequence converges to 0 in $B_{0}+B_{1}$ and $B$ is an intermediate space. Thus we deduce that in this case (iii) cannot hold. This shows that (iii) $\Rightarrow$ (i) and completes the proof.

\section{Some results for other operator ideals}

The class of compact operators between Banach spaces is an injective surjective closed operator ideal in the sense of Pietsch. It is therefore natural to investigate whether the results of $\S 3$ are valid for other operator ideals having similar properties.

Let us recall (cf. [8, p. 131] and [17, p. 45]) that an operator ideal $\mathcal{I}$ is a method of ascribing to each pair $(X, Y)$ of Banach spaces, a linear subspace $\mathcal{I}(X, Y)$ of $\mathcal{L}(X, Y)$ such that

(i) $\mathcal{I}(X, Y)$ contains all rank one operators $x^{*} \otimes y$ for all $x^{*} \in X^{*}$ and $y \in Y$; and

(ii) for all Banach spaces $X, Y, E, F$, whenever $U \in \mathcal{L}(E, X), T \in \mathcal{I}(X, Y)$ and $S \in \mathcal{L}(Y, F)$, then the composed operator $S T U \in \mathcal{I}(E, F)$. 
The ideal $\mathcal{I}$ is closed if $\mathcal{I}(X, Y)$ is a closed subspace of $\mathcal{L}(X, Y)$ with respect to the usual operator norm topology, for all Banach spaces $X$ and $Y$.

For each Banach space $A$ we let $Q_{A}$ denote the canonical surjection $Q_{A}: \ell_{1}\left(U_{A}\right) \rightarrow A$ defined by

$$
Q_{A}\left(\left\{\lambda_{x}\right\}_{x \in U_{A}}\right)=\sum_{x \in U_{A}} \lambda_{x} x
$$

and $J_{A}$ denote the canonical isometric embedding $J_{A}: A \rightarrow \ell_{\infty}\left(U_{A^{*}}\right)$ defined by

$$
J_{A} a=\{\langle f, a\rangle\}_{f \in U_{A^{*}}} .
$$

The operator ideal $\mathcal{I}$ is said to be injective (respectively, surjective) if whenever $T \in$ $\mathcal{L}(A, B)$ and in addition $J_{B} T \in \mathcal{I}\left(A, \ell_{\infty}\left(U_{B^{*}}\right)\right)$ (respectively, $T Q_{A} \in \mathcal{I}\left(\ell_{1}\left(U_{A}\right), B\right)$ ), then it follows that $T \in \mathcal{I}(A, B)$ (cf. [17, pp. 70-75]).

Apart from the class of compact operators, other examples of injective surjective closed ideals are the classes of weakly compact operators, Rosenthal operators, Banach-Saks operators and dual Radon-Nikodým operators. The class of strictly singular operators is an injective closed ideal which is not surjective, while the class of strictly cosingular operators is a surjective closed ideal which is not injective. We refer to [17] and also to $[\mathbf{8}]$ and $[\mathbf{1 2}]$ for more details about operator ideals.

There are two functionals, introduced, respectively, by Astala and by Tylli, which can be used to measure the extent to which a given operator $T \in \mathcal{L}(A, B)$ fails to belong to a given operator ideal $\mathcal{I}$. We shall now briefly recall their definitions and some of their main properties. We refer to [6] for relevant references and further details.

The outer measure of $T \in \mathcal{L}(A, B)$ is denoted by $\gamma_{\mathcal{I}}(T)$ or $\gamma_{\mathcal{I}}\left(T_{A, B}\right)$ and is the infimum of all positive numbers $\sigma$ such that $T\left(U_{A}\right) \subset \sigma U_{B}+R\left(U_{Z}\right)$ for some Banach space $Z$ and some operator $R \in \mathcal{I}(Z, B)$. The inner measure of the same operator is denoted by $\beta_{\mathcal{I}}(T)$ or $\beta_{\mathcal{I}}\left(T_{A, B}\right)$ and is the infimum of all positive numbers $\sigma$ such that for some Banach space $Z$ and some operator $R \in \mathcal{I}(A, Z)$ the inequality

$$
\|T x\|_{B} \leqslant \sigma\|x\|_{A}+\|R x\|_{Z}
$$

holds for all $x \in A$.

It is known that

if $\mathcal{I}$ is surjective and closed, then $\gamma_{\mathcal{I}}\left(T_{A, B}\right)=0$ if and only if $T \in \mathcal{I}(A, B)$,

and, analogously,

if $\mathcal{I}$ is injective and closed, then $\beta_{\mathcal{I}}\left(T_{A, B}\right)=0$ if and only if $T \in \mathcal{I}(A, B)$.

In the particular case where $\mathcal{I}=\mathcal{K}$, the ideal of compact operators, then $\gamma_{\mathcal{K}}\left(T_{A, B}\right)$ coincides with the measure of non-compactness of $T$ and $\beta_{\mathcal{K}}\left(T_{A, B}\right)$ is the infimum of all $\eta>0$ such that there exists a subspace $M$ of $A$ with finite codimension such that $\|T x\|_{B} \leqslant \eta\|x\|_{A}$ for all $x \in M$.

The following theorem is an abstract version of Theorem 3.1. In its proof we use some techniques that were developed in $[\mathbf{6}]$. 
Theorem 4.1. Let $\bar{A}=\left(A_{0}, A_{1}\right)$ be a Banach couple, let $A$ be an intermediate space with respect to $\bar{A}$ and let $B$ be another Banach space. Let $\mathcal{I}$ be an operator ideal. Then, for each $T \in \mathcal{L}(\bar{A}, B)$ :

(a) if $\gamma_{\mathcal{I}}\left(T_{A_{0}, B}\right)=0$, then

$$
\gamma_{\mathcal{I}}\left(T_{A, B}\right) \leqslant \gamma_{\mathcal{I}}\left(T_{A_{1}, B}\right) \cdot \lim _{t \rightarrow \infty} \frac{\psi(t, A, \bar{A})}{t}
$$

(b) if $\gamma_{\mathcal{I}}\left(T_{A_{1}, B}\right)=0$, then

$$
\gamma_{\mathcal{I}}\left(T_{A, B}\right) \leqslant \gamma_{\mathcal{I}}\left(T_{A_{0}, B}\right) \cdot \lim _{t \rightarrow 0} \psi(t, A, \bar{A})
$$

and

(c) if $\gamma_{\mathcal{I}}\left(T_{A_{i}, B}\right)>0$ for $i=0,1$, then

$$
\gamma_{\mathcal{I}}\left(T_{A, B}\right) \leqslant 2 \gamma_{\mathcal{I}}\left(T_{A_{0}, B}\right) \psi\left(\frac{\gamma_{\mathcal{I}}\left(T_{A_{1}, B}\right)}{\gamma_{\mathcal{I}}\left(T_{A_{0}, B}\right)}, A, \bar{A}\right) .
$$

Proof. By the definition of $\gamma\left(T_{A_{i}, B}\right)$, for $i=0,1$ and for each $\epsilon>0$ there exist Banach spaces $Z_{i}$ and operators $S_{i} \in \mathcal{I}\left(Z_{i}, B\right)$ such that

$$
T\left(U_{A_{i}}\right) \subset\left(\epsilon+\gamma_{\mathcal{I}}\left(T_{A_{i}, B}\right)\right) U_{B}+S_{i}\left(U_{Z_{i}}\right) .
$$

Now, for each choice of positive numbers $\delta$ and $t$ and each $a \in U_{A}$, there exists a decomposition $a=a_{0}+a_{1}$ with $a_{i} \in A_{i}$ and $a_{i} \in(\delta+\psi(t)) t^{-i} U_{A_{i}}$ for $i=0,1$. From this and (4.3) it follows that

$$
\begin{aligned}
T\left(U_{A}\right) & \subset(\delta+\psi(t))\left[T\left(U_{A_{0}}\right)+\frac{1}{t} T\left(U_{A_{1}}\right)\right] \\
& \subset(\delta+\psi(t))\left[\epsilon+\gamma_{\mathcal{I}}\left(T_{A_{0}, B}\right)+\frac{\epsilon}{t}+\frac{1}{t} \gamma_{\mathcal{I}}\left(T_{A_{1}, B}\right)\right] U_{B}+S_{0}^{\prime}\left(U_{Z_{0}}\right)+S_{1}^{\prime}\left(U_{Z_{1}}\right),
\end{aligned}
$$

where $S_{0}^{\prime}=(\delta+\psi(t)) S_{0}$ and $S_{1}^{\prime}=(1 / t)(\delta+\psi(t)) S_{1}$ are operators belonging to $\mathcal{I}\left(Z_{0}, B\right)$ and $\mathcal{I}\left(Z_{1}, B\right)$, respectively. Let $Z$ be the Banach space $Z=Z_{0} \oplus Z_{1}$ with norm $\|(x, y)\|_{Z}=$ $\max \left\{\|x\|_{Z_{0}},\|y\|_{Z_{1}}\right\}$ and define $S: Z \rightarrow B$ by $S(x, y)=S_{0}^{\prime} x+S_{1}^{\prime} y$. Then clearly $S\left(U_{Z}\right)=$ $S_{0}^{\prime}\left(U_{Z_{0}}\right)+S_{1}^{\prime}\left(U_{Z_{1}}\right)$, and, furthermore, using the projection operators from $Z$ onto $Z_{0}$ and $Z_{1}$, and the definition of operator ideals, we have that $S \in \mathcal{I}(Z, B)$. Consequently,

$$
\gamma_{\mathcal{I}}\left(T_{A, B}\right) \leqslant \psi(t)\left[\gamma_{\mathcal{I}}\left(T_{A_{0}, B}\right)+\frac{1}{t} \gamma_{\mathcal{I}}\left(T_{A_{1}, B}\right)\right], \quad \text { for all } t>0 .
$$

This inequality implies the three cases of the theorem by the same immediate arguments as were used to obtain the three cases of Theorem 3.1 from equation (3.2).

We now consider an abstract version of Theorem 3.2. Note, however, that in the particular case $\mathcal{I}=\mathcal{K}$, it yields a result different from Theorem 3.2, since here we deal with the inner rather than outer measure. (Also the factors of 2 that appear in Theorem 3.2 do not appear here.) 
Theorem 4.2. Let $\bar{B}=\left(B_{0}, B_{1}\right)$ be a Banach couple, let $B$ be an intermediate space with respect to $\bar{B}$ and let $A$ be another Banach space. Let $\mathcal{I}$ be an operator ideal. Then, for each operator $T \in \mathcal{L}(A, \bar{B})$ :

(a) if $\beta_{\mathcal{I}}\left(T_{A, B_{0}}\right)=0$, then

$$
\beta_{\mathcal{I}}\left(T_{A, B}\right) \leqslant \beta_{\mathcal{I}}\left(T_{A, B_{1}}\right) \cdot \lim _{t \rightarrow 0} \frac{t}{\rho(t, B, \bar{B})} ;
$$

(b) if $\beta_{\mathcal{I}}\left(T_{A, B_{1}}\right)=0$, then

$$
\beta_{\mathcal{I}}\left(T_{A, B}\right) \leqslant \beta_{\mathcal{I}}\left(T_{A, B_{0}}\right) \cdot \lim _{t \rightarrow \infty} \frac{1}{\rho(t, B, \bar{B})} ;
$$

and

(c) if $\beta_{\mathcal{I}}\left(T_{A, B_{i}}\right)>0$ for $i=0,1$, then

$$
\beta_{\mathcal{I}}\left(T_{A, B}\right) \leqslant \frac{\beta_{\mathcal{I}}\left(T_{A, B_{0}}\right)}{\rho\left(\beta_{\mathcal{I}}\left(T_{A, B_{0}}\right) / \beta_{\mathcal{I}}\left(T_{A, B_{1}}\right), B, \bar{B}\right)} .
$$

Proof. In view of the definition of $\beta_{\mathcal{I}}\left(T_{A, B_{i}}\right)$, given any $\epsilon>0$, there are Banach spaces $Z_{i}$ and operators $S_{i} \in \mathcal{I}\left(A, Z_{i}\right)$ such that

$$
\|T a\|_{B_{i}} \leqslant\left(\epsilon+\beta_{\mathcal{I}}\left(T_{A, B_{i}}\right)\right)\|a\|_{A}+\left\|S_{i} a\right\|_{Z_{i}}, \quad \text { for all } a \in A, \quad i=0,1 .
$$

Since $T a \in B_{0} \cap B_{1}$ and $\|b\|_{B} \leqslant(1 / \rho(t, B, \bar{B})) J(t, b ; \bar{B})$ for all $b \in B_{0} \cap B_{1}$ and all $t>0$, we obtain that

$$
\begin{aligned}
\|T a\|_{B} & \leqslant \frac{1}{\rho(t)} J(t, T a) \\
& \leqslant \frac{1}{\rho(t)} \max \left\{\left(\epsilon+\beta_{\mathcal{I}}\left(T_{A, B_{0}}\right)\right), t\left(\epsilon+\beta_{\mathcal{I}}\left(T_{A, B_{1}}\right)\right)\right\}\|a\|_{A}+\left\|S_{0}^{\prime} a\right\|_{Z_{0}}+\left\|S_{1}^{\prime} a\right\|_{Z_{1}},
\end{aligned}
$$

where $S_{0}^{\prime}=(1 / \rho(t)) S_{0}$ and $S_{1}^{\prime}=(t / \rho(t)) S_{1}$. Let $Z$ be the Banach space $Z=Z_{0} \oplus Z_{1}$ with norm $\|(x, y)\|_{Z}=\|x\|_{Z_{0}}+\|y\|_{Z_{1}}$ and consider the operator $S: A \rightarrow Z$ defined by $S a=\left(S_{0}^{\prime} a, S_{1}^{\prime} a\right)$. Then, using the ideal properties of $\mathcal{I}$ and the canonical embeddings of $Z_{0}$ and $Z_{1}$ into $Z$, we see that $S \in \mathcal{I}(A, Z)$ and we have

$$
\|T a\|_{B} \leqslant \frac{1}{\rho(t)} \max \left\{\left(\epsilon+\beta_{\mathcal{I}}\left(T_{A, B_{0}}\right)\right), t\left(\epsilon+\beta_{\mathcal{I}}\left(T_{A, B_{1}}\right)\right)\right\}\|a\|_{A}+\|S a\|_{Z}, \quad \text { for all } a \in A .
$$

This implies that

$$
\beta_{\mathcal{I}}\left(T_{A, B}\right) \leqslant \frac{1}{\rho(t)} \max \left\{\beta_{\mathcal{I}}\left(T_{A, B_{0}}\right), t \beta_{\mathcal{I}}\left(T_{A, B_{1}}\right)\right\}, \quad \text { for all } t>0,
$$

and we can conclude the proof in the same way as at the end of the proof of Theorem 3.2. 
Suppose that $T: \bar{A} \rightarrow B$ and $T \in \mathcal{I}\left(A_{1}, B\right)$ for some operator ideal $\mathcal{I}$. For which intermediate spaces or rank-one interpolation spaces $A$ do we necessarily have that $T \in$ $\mathcal{I}(A, B)$ ? Under what circumstances can it happen that $T \notin \mathcal{I}(A, B)$ ?

The preceding two theorems enable us to find answers to these questions and the corresponding questions for operators $T: A \rightarrow \bar{B}$ with $T \in \mathcal{I}\left(A, B_{1}\right)$. These answers take the form of analogues of Theorems 3.9 and 3.10 and Corollaries 3.11 and 3.12, where the ideal $\mathcal{K}$ of compact operators is replaced by an appropriate more general operator ideal $\mathcal{I}$.

First we state a result that incorporates the analogues of Theorem 3.9 and Corollary 3.11. Here, we need the ideal to be surjective and closed so that we can use property (4.1). The proof of the theorem can then follow exactly the same path as its predecessor theorem and corollary, except that the inner measure $\gamma_{\mathcal{I}}$ now plays the former role of the measure of non-compactness.

Theorem 4.3. Let $\bar{A}=\left(A_{0}, A_{1}\right)$ be a Banach couple, let $A$ be a rank-one interpolation space with respect to $\bar{A}$ and let $B$ be another Banach space. Let $\mathcal{I}$ be a surjective closed operator ideal and let $T: \bar{A} \rightarrow B$ be a linear operator such that $T \in \mathcal{I}\left(A_{1}, B\right)$. Then at least one of the following conditions must hold.

(i) $T \in \mathcal{I}(A, B)$.

(ii) $A_{0}^{\circ} \hookrightarrow A$.

If, in addition, the couple $\bar{A}$ satisfies $A_{0}^{\circ}=A_{0}$, then $T \in \mathcal{I}(A, B)$ if and only if at least one of the following conditions hold.

(i') $\lim _{t \rightarrow 0} \psi(t, A, \bar{A})=0$.

(ii') $T \in \mathcal{I}\left(A_{0}, B\right)$.

Now we turn to a theorem that contains the analogues of Theorem 3.10 and Corollary 3.12. Here, the ideal must be taken to be injective and closed so that the proof can use property (4.2) and the outer measure $\beta_{\mathcal{I}}$ in place of the measure of non-compactness.

Theorem 4.4. Let $\bar{B}=\left(B_{0}, B_{1}\right)$ be a Banach couple, let $B$ be a rank-one interpolation space with respect to $\bar{B}$, and let $A$ be another Banach space. Let $\mathcal{I}$ be an injective closed operator ideal, and suppose that $T \in \mathcal{L}(A, \bar{B})$ satisfies $T \in \mathcal{I}\left(A, B_{1}\right)$. Then at least one of the following conditions must hold.

(i) $T \in \mathcal{I}(A, B)$.

(ii) $B \hookrightarrow B_{0}^{\backsim}$.

If, in addition, we have $B_{0}^{\backsim}=B_{0}$, then $T \in \mathcal{I}(A, B)$ if and only if at least one of the following conditions hold.

(i') $\lim _{t \rightarrow \infty} \rho(t, B, \bar{B})=\infty$.

(ii') $T \in \mathcal{I}\left(A, B_{0}\right)$. 
Theorems 4.1 and 4.2 immediately lead to stronger versions of Propositions 1.6 and 1.7 in Heinrich [12], i.e. if $\mathcal{I}$ is closed and, respectively, surjective or injective, and if $T \in$ $\mathcal{I}\left(A_{1}, B\right)$ or, respectively, $T \in \mathcal{I}\left(A, B_{1}\right)$, and if (1.1) or, respectively, (1.2) holds, then Theorem 4.1 or, respectively, Theorem 4.2 immediately gives us that $T \in \mathcal{I}(A, B)$. Applying these results to the ideals of strictly singular operators and strictly cosingular operators, we obtain new information about the behaviour under interpolation of these operator ideals which complements the results established by Beucher in $[\mathbf{3}, \S 2]$.

In the cases where $A$ or $B$, respectively, are merely intermediate spaces rather than rank-one interpolation spaces we apparently, in general, do not have analogues of Theorems 3.15 and 3.17 .

Acknowledgements. F.C. was supported in part by Dirección General de Enseňanza Superior (PB97-0254). M.C. was supported by the Fund for Promotion of Research at the Technion. P.M. was supported by Progama Praxis XXI da Fundaçăo para a Ciência e Tecnologia (BD-11466-97) and by Instituto Politécnico de Leiria. We are grateful to Evgeniy Pustylnik for drawing our attention to an interesting example and also for a number of other helpful suggestions and remarks.

\section{References}

1. C. Bennett And R. Sharpley, Interpolation of operators (Academic Press, New York, 1988).

2. J. BERGH AND J. LÖFstRÖM, Interpolation spaces. An introduction, Grundlehren der Mathematische Wissenschaften, vol. 223 (Springer, Berlin, 1976).

3. O. J. Beucher, On interpolation of strictly (co-)singular linear operators, Proc. R. Soc. Edinb. A 112 (1989), 263-269.

4. Y. BRUdny and N. KRUGlJak, Interpolation functors and interpolation spaces, vol. 1 (North Holland, Amsterdam, 1991).

5. F. Cobos, T. Kühn And T. SchonbeK, One-sided compactness results for AronszajnGagliardo functors, J. Funct. Analysis 106 (1992), 274-313.

6. F. Cobos, A. Manzano And A. Martínez, Interpolation theory and measures related to operator ideals, Q. J. Math. Oxford (2) 50 (1999), 401-416.

7. F. Cobos And J. PeEtre, Interpolation of compactness using Aronszajn-Gagliardo functors, Israel J. Math. 68 (1989), 220-240.

8. J. Diestel, H. Jarchow and A. Tonge, Absolutely summing operators, Cambridge Studies in Advanced Mathematics, vol. 43 (Cambridge University Press, 1995).

9. A. A. DMitriev, The interpolation of one-dimensional operators, Voronež Gos. Univ. Trudy Naučn.-Issled. Inst. Mat. VGU Vyp. 11 Sb. Statej Funkcional. Anal. i Prilozen 11 (1973), 31-43 (in Russian).

10. E. Gagliardo, A unified structure in various families of function spaces. Compactness and closure theorems, in Proc. Int. Symp. Linear Spaces, Jerusalem 1960, pp. 237-241 (Pergamon, Oxford, 1961).

11. G. H. Hardy And J. E. Littlewood, Some properties of fractional integrals (I), Math. Z. 27 (1928), 565-606.

12. S. HeInRICH, Closed operator ideals and interpolation, J. Funct. Analysis 35 (1980), 397-411.

13. M. A. Krasnoselskit, P. P. Zabreiko, E. I. Pustylnik and P. E. Sobolevskit, Integral operators in spaces of summable functions (Noordhoff, Leyden, 1976). 
14. J. L. Lions and J. Peetre, Sur une classe d'espaces d'interpolation, Inst. Hautes Etudes Sci. Publ. Math. 19 (1964), 5-68.

15. M. MastyŁo, On interpolation of compact operators, Funct. Approx. Comment. Math. 26 (1998), 293-311.

16. A. Persson, Compact linear mappings between interpolation spaces, Ark. Mat. 5 (1964), 215-219.

17. A. Pietsch, Operator ideals (North-Holland, Amsterdam, 1980).

18. E. I. PUSTYLnik, On optimal interpolation and some interpolation properties of Orlicz spaces, Soviet. Math. Dokl. 27 (1983), 333-336. (Translation from Dokl. Akad. Nauk SSSR 269 (1983), 292-295.)

19. E. I. PUSTYlnik, Embedding functions and their role in interpolation theory, Abstract Appl. Analysis 1 (1996), 305-325.

20. M. F. TEIXEIRA AND D. E. EDmunds, Interpolation theory and measures of non-compactness, Math. Nachr. 104 (1981), 129-135. 